\title{
A importância da Vacina Pneumocócica Polissacarídica 23-Valente (PPSV23) na prevenção de doenças pneumocócicas no Brasil
}

The importance of the 23-Valent Pneumococcal Polysaccharide Vaccine (PPSV23) in the prevention of pneumococcal diseases in Brazil

La importancia de la Vacuna Polisacárida Neumocócica 23-Valente (PPSV23) en la prevención de enfermedades neumocócicas en Brasil

Luan Daniel Silva Ferreira ${ }^{1 *}$, Laura Cristina Correa Costa ${ }^{1}$, Erlane Paiva Russo ${ }^{1}$, Priscila Cerdeira Coelho de Sousa1, Bruno Pantoja Gadelha ${ }^{1}$, Analice Ramos Fernandes ${ }^{1}$, Fabiolla da Silva dos Santos ${ }^{1}$, Rosiane Paiva Russo ${ }^{2}$, Sérgio Carlos dos Santos Gomes ${ }^{3}$.

\begin{abstract}
RESUMO
Objetivo: Avaliar a importância da Vacina Pneumocócica Polissacarídica 23-Valente (PPSV23) na prevenção das doenças pneumocócicas no Brasil. Revisão bibliográfica: Streptococcus pnemoniae causa infecção e afeta principalmente as crianças, pessoas acima de 65 anos de idade, imunodeprimidos e doentes crônicos. Sob essa perspectiva, as doenças relacionadas ao $S$. pnemoniae compõem uma problemática significativa para a saúde pública geral, além disso, o aumento exacerbado do número de cepas resistentes a antibióticos acentua o impasse. No Brasil, a pneumonia se destaca como a segunda causa de morte. A mortalidade por pneumonia é maior nos extremos de idade, chegando a 8,4\% em menores de 5 anos e $61 \%$ em indivíduos com 70 anos ou mais. É importante notar que a PPSV23 é considerada uma vacina custo-efetiva. Considerações finais: $O$ uso da vacina PPSV23, no estudo, demonstrou a capacidade de conter o impacto crescente da doença, e também reduzir a resistência aos antibióticos, evitando o surgimento de cepas resistentes, pelo uso diminuto desses antibióticos. Por fim, faz-se necessário mais estudos desse cunho, para que se possa reunir informações e associá-la, de maneira a melhor compreender os impactos que a vacinação contra as doenças pneumocócicas possa trazer para a sociedade.
\end{abstract}

Palavras-chave: Infecção, Saúde pública, Doença, Mortalidade.

\begin{abstract}
Objective: To evaluate the importance of the 23-Valent Pneumococcal Polysaccharide Vaccine (PPSV23) in the prevention of pneumococcal diseases in Brazil. Bibliografic review: Streptococcus pnemoniae causes infection and mainly affects children, people over 65 years of age, immunocompromised and chronically ill. From this perspective, diseases related to $S$. pnemoniae are a significant problem for general public health, in addition, the exacerbated increase in the number of antibiotic-resistant strains accentuates the impasse. In Brazil, pneumonia stands out as the second leading cause of death. Mortality from pneumonia is higher at the extremes of age, reaching $8.4 \%$ in children under 5 years of age and $61 \%$ in individuals aged 70 years and over. It is important to note that PPSV23 is considered a cost-effective vaccine. Final considerations: The use of the PPSV23 vaccine, in the study, demonstrated the ability to contain the growing impact of the disease, and also to reduce antibiotic resistance, preventing the emergence of resistant strains, due to the low use of these antibiotics. Finally, more studies of this nature are needed, so that information can be gathered and associated, in order to better understand the impacts that vaccination against pneumococcal diseases can bring to society.
\end{abstract}

Keywords: Infection, Public health, Disease, Mortality.

1 Escola Superior da Amazônia (ESAMAZ), Belém - PA. *E-mail: luan.ferreirabio@gmail.com

${ }^{2}$ Faculdades Integradas Norte do Paraná (UNOPAR), Barcarena - PA.

3 Universidade Anhanguera, Belém - PA.

SUBMETIDO EM: 10/2021

ACEITO EM: 10/2021

PUBLICADO EM: 10/2021 


\section{RESUMEN}

Objetivo: Evaluar la importancia de la Vacuna Polisacárida Neumocócica 23-Valente (PPSV23) en la prevención de enfermedades neumocócicas en Brasil. Revisión bibliográfica: Streptococcus pnemoniae causa infección y afecta principalmente a niños, personas mayores de 65 años, inmunodeprimidos y enfermos crónicos. Desde esta perspectiva, las enfermedades relacionadas con $S$. pnemoniae son un problema importante para la salud pública en general, además, el aumento exacerbado del número de cepas resistentes a los antibióticos acentúa el impasse. En Brasil, la neumonía se destaca como la segunda causa principal de muerte. La mortalidad por neumonía es mayor en los extremos de edad, alcanzando el 8,4\% en niños menores de 5 años y el $61 \%$ en personas de 70 años o más. Es importante señalar que la PPSV23 se considera una vacuna rentable. Consideraciones finales: El uso de la vacuna PPSV23, en el estudio, demostró la capacidad de contener el impacto creciente de la enfermedad, y también de reducir la resistencia a los antibióticos, evitando la aparición de cepas resistentes, debido al bajo uso de estos antibióticos. Finalmente, se necesitan más estudios de esta naturaleza, de manera que se pueda recopilar y asociar información, con el fin de comprender mejor los impactos que la vacunación contra las enfermedades neumocócicas puede traer a la sociedad.

Palabras clave: Infección, Salud pública, Enfermedad, Mortalidad.

\section{INTRODUÇÃO}

Nos países desenvolvidos, as mortes substanciais são em maioria por infecções respiratórias agudas, acarretando aproximadamente 4 milhões de falecidos mundialmente (FERKOL T e SCHRAUFNAGEL D, 2014). Um grupo de doenças causadas pela bactéria Streptococcus pneumoniae (pneumococo), são denominadas de doenças pneumocócicas e correspondem a pneumonia, septicemia, meningite, otite média aguda e sinusite (BAHIA L, et al., 2013).

Esta bactéria e caracterizada por fazer parte do grupo Mitis dos estreptococos, e 12 espécies filogeneticamente relacionadas, filogenia baseada através do sequenciamento do rRNA 16S. Várias espécies que pertencem a esse grupo são comensais, e abrigam-se no aparelho respiratório superior, sendo chamadas vulgarmente de estreptococos viridans (MIRANDA JMD, 2011).

De acordo com o Centro de Controle e Prevenção de Doenças (CDC), A transmissão de S. pneumoniae se dá do contato direto entre pessoas, através de gotículas respiratórias e por autoinoculação, advinda de pessoas portadoras da bactéria no trato respiratório superior (CDC, 2019). Segundo a Organização Mundial de Saúde (OMS), as infeções do trato respiratório inferior constituem a doença transmissível mais mortal e em 2015 causaram 3,2 milhões de mortes em todo o mundo (OMS, 2021).

$S$ pneumoniae foi a principal causa de morbidade e mortalidade das doenças em todo o mundo, contribuindo para 1189937 mortes, que foram mais do que todas as outras etiologias combinadas em 2016. O programa de imunização é destinado a adultos mais velhos que com idade $\geq 65$ anos, e aqueles que com 60-64 e têm atividade diária extremamente restrita devido a um distúrbio no coração, rim, função respiratória ou imunológica função devido à infecção pelo HIV (MURAKAMI Y, et al., 2016).

Mundialmente, o S. pneumoniae se tornou o motivo primordial para infecções de pneumonia, dados presumem que entre 30 a $50 \%$ do total de ocorrência sejam relatados como Pneumonia Adquirida na Comunidade (PAC), levando a internações (MARQUES SC, 2016). É também um dos principais responsáveis pelo aumento da morbidade e mortalidade da população acima dos 65 anos e causa de uma ampla variedade de doenças invasivas, como pneumonia bacteriana, septicemia e meningite (ALVAREZ CE, et al., 2017; HUIJTS SM, et al., 2017).

Em indivíduos com idade igual ou superior a 60 anos no continente americano, a pneumonia oscila entre as posições de primeiro e terceiro lugar no ranking das causas de mortalidade, e de acordo com o CDC, dados de 2009 (BLAMEY R, 2014).

A doença pneumocócica é uma causa comum de morbidade e mortalidade em todo o mundo. A OMS relatou um total de 1,6 milhão de mortes causadas globalmente por Streptococcus pneumoniae a cada ano 
(OMS, 2021). Desse modo, é importante que os países realizem vigilância contínua da distribuição de sorotipos e das taxas de incidência para detectar e relatar diferenças regionais e monitorar o efeito da vacinação (ZINTGRAFF J, et al., 2020).

As vacinas trazem vantagem significativa com benefícios potenciais associados ao "boosting" natural. Evitam o aumento da densidade do transporte de $S$. pneumoniae, mantendo-o abaixo do limite da doença. Sob essa perspectiva, as vacinas em geral, auxiliam na resposta imune mais forte, quando analisada a memória induzida provocado pela vacina (PICHICHERO ME, et al., 2016).

A vacina pneumocócica polissacarídica 23-valente (PPSV23) é eficaz na prevenção da pneumonia causada por cepas de $S$. pneumoniae em idosos $\geq 65$ anos. Foi relatado que os seguintes determinantes epidemiológicos estão associados à cobertura do PPSV23: idade, sexo, educação, renda, estado civil, estrutura familiar, saúde autoanalisada, saúde, alfabetização, tabagismo, médico de clínica geral, doenças de alto risco para pneumonia, incluindo diabetes, doenças cardíacas e doenças respiratórias crônicas (CHEN $\mathrm{CH}$, et al., 2018; GATWOOD J, et al., 2020).

Desse modo, o presente estudo teve o objetivo de avaliar a importância da Vacina Pneumocócica Polissacarídica 23-Valente (PPSV23) na prevenção das doenças pneumocócicas no Brasil, com intuito de gerar dados relevantes para auxiliar nas futuras tomadas de decisões, em relação ao combate e erradicação da infecção.

\section{REVISÃO BIBLIOGRAFICA}

\section{Epidemiologia}

$\mathrm{Na}$ população maior de 18, duas vacinas pneumocócicas são administradas, com intuito de prevenir infecções por S. pneumoniae, são a PPSV23 e a Vacina Pneumocócica Conjugada PCV13 (COSTA RP, et al., 2016). Ainda existe uma relevante discussão em torno da vacinação dos idosos e como a estratégia é executada, principalmente nos países desenvolvimento (FALKENHORST G, et al., 2017).

Nos Estados Unidos, considerando todas as faixas etárias, a incidência global de doença pneumocócica invasiva (DPI) foi de 12,8 casos por 100 habitantes em 2010. Na Europa, apesar das taxas variarem bastantes entre os diferentes países, relatou-se uma taxa média em 24 países europeus de 4,3 casos por 100 mil indivíduos (VILA-CORCOLES A e OCHOA-GONDAR O, 2013). De acordo com a OMS a doença apresenta maiores taxas de incidência e de mortalidade em países subdesenvolvidos (JIANG Y, et al., 2014).

Nos Estados Unidos, foi licenciada uma vacina polissacarídica contendo 14 sorotipos capsulares, a qual em no ano de 1983 foi substituída pela atual PPSV23 que é composta por $25 \mu \mathrm{g}$ de antígenos polissacarídicos capsulares purificados de 23 sorotipos causadores de DPI (MOBERLEY S, et al., 2013). Ao observar o Plano Nacional Australiano, a vacinação antipneumocócica com PPSV23 é aconselhada em indivíduos idosos (CORREIA S, 2013).

Um dos maiores números de casos globais registrados é no Brasil. Esses dados foram obtidos do Sistema de Informação sobre Mortalidade do Departamento de Tecnologia da Informação do Sistema Único de Saúde (SIM/DATASUS), no intervalo de 1996 a 2002, quando observadas as infecções respiratórias a pneumonia alcançou incríveis 37\% das mortes (NUNES BP, et al., 2017).

Cabe notar que outros países enfrentam situação semelhante de baixa frequência de vacinação pneumocócica entre indivíduos com indicação clínica da vacina (PALLOTTA A e REHM SJ, 2016) e que é de consenso geral a necessidade do desenvolvimento de estratégias que visem favorecer 0 aumento da cobertura vacinal para vários imunobiológicos (GONZÁLEZ DFR, et al., 2015).

No Brasil, dentro do grupo das doenças respiratórias, a pneumonia se destaca como a segunda causa de morte, ficando também em segundo lugar em relação às causas de internação. A mortalidade por pneumonia é maior nos extremos de idade, chegando a 8,4\% em menores de 5 anos e $61 \%$ em indivíduos com 70 anos ou mais (MARTINS JP, et al., 2019). 
A meningite bacteriana é menos comum que do a pneumonia, mas possui alto risco de sequela e letalidade. No Brasil, de 2000 a 2008, a Meningite Pneumocócica (MP) representou $11 \%$ de todas as meningites bacterianas, com incidência de 9,5 casos por 100 mil habitantes em crianças com menos de um ano. Dados oficiais do país, do mesmo período, registraram 7.129.291 hospitalizações associadas a complicações de PAC, tendo o Streptococcus pneumoniae como o principal agente etiológico (CAIERAO J, et al., 2014).

Além da prevalência significativa da doença pneumocócica, outro problema importante é a resistência bacteriana. De acordo com o CDC, em Atlanta, Estados Unidos, em 30\% dos casos graves da doença, a bactéria é totalmente resistente a um ou mais antibióticos (ALEXANDRE RF, et al., 2016).

A educação e o apoio contínuo ao autocuidado do paciente são essenciais para prevenir complicações agudas e reduzir o risco de complicações a longo prazo, assim como prevenir o risco de doenças associadas, mantendo assim a qualidade de vida destes pacientes (AMERICAN DIABETES ASSOCIATION, 2019).

\section{Sorotipos}

A prevalência de cada sorotipo nas estirpes de doenças não invasivas e invasivas é diferente e só um número restrito é responsável pela maioria destas últimas (MIRANDA JMD, 2011).

Existem mais de 90 sorotipos de pneumococo que podem causar doenças infecciosas e a Organização Mundial de Saúde (OMS) recomenda o uso de vacinas polissacarídicas contendo os seguintes 23 sorotipos de pneumococos: 1, 2, 3, 4, 5, 6B, 7F, 8 ,9N, 9V, 10A, 11A, 12F, 14, 15B, 17F, 18C,19A, 19F, 20, 22F, $23 \mathrm{~F} \mathrm{e}$ 33F, por serem as cepas presentes entre 85 a $90 \%$ dos casos de DPI entre adultos nos EUA e outros países industrializados (MARTINS SP, 2019).

Dados sobre a prevalência dos sorotipos no Brasil podem ser obtidos de relatórios do Sistema de Redes de Vigilância dos Agentes Responsáveis por Pneumonia e Meningites Bacterianas (SIREVA). Essa rede de vigilância laboratorial passiva foi instituída em 1993 com patrocínio da organização Pan-Americana da Saúde (OPAS) e do Ministério da Saúde (MS), com o propósito de criar um banco de material biológico associado a uma informação epidemiológica e de carga da doença (como sorotipagem e resistência in vitro dos patógenos), para auxiliar as autoridades nacionais na tomada de decisão (INFORME REGIONAL DE SIREVA II, 2011).

Embora uma grande variedade de sorotipos cause doenças não invasivas, como otite média e sinusite, os sorotipos 1, 5, 6A, 6B, 14, 19F e 23F são causas comuns de Doenças Invasivas Pneumocócicas (DIP) globalmente em crianças $<5$ anos de idade. Além disso, alguns sorotipos como $6 \mathrm{~B}, 9 \mathrm{~V}, 14,19 \mathrm{~A}, 19 \mathrm{~F}$ e $23 \mathrm{~F}$ são mais prováveis do que outros para serem associados à resistência aos medicamentos (OMS, 2021).

Além da idade, a presença de comorbilidades crónicas (doença pulmonar, cardiovascular, renal, hepática e diabetes mellitus), situações de imunodeficiência ou imunocompromisso e certos estilos de vida (como o tabagismo ou o alcoolismo) podem aumentar o risco de doença pneumocócica. Deste modo, tendo em conta a presença de imunossenescência, fenómeno explicado mais à frente, e a prevalência destas comorbilidades crónicas nos idosos, estes constituem um grupo com alto risco de contrair doença pneumocócica (REIS SAA, 2015).

\section{Vacina Pneumocócica Polissacarídea 23-Valente (PPSV23)}

A primeira forma de vacina contra o pneumococo foi desenvolvida em 1911, criada após 30 anos do isolamento dessa bactéria. Ao passar dos anos, com base nos estudos com vacinas incluindo dois ou mais sorotipos, duas vacinas hexavalentes foram comercializadas a partir do final do ano de 1940. Todavia, em um primeiro momento, frente ao advento dos antibióticos, houve diminuição da crença nas vacinas, acreditandose que a antibioticoterapia seria totalmente eficaz no combate às infecções pneumocócicas (MARTINS SP, 2019).

A vacina PPSV23 promove resposta imunológica T-independente, de curta duração, e não induz a memória imunológica principalmente quando utilizada em menores de 2 anos de idade devido a seu sistema 
imunológico ainda imaturo. Por outro lado, acima dessa idade, a vacina induz o dobro do crescimento de anticorpos específicos aos sorotipos presentes na vacina dentro de duas ou três semanas na população acima dessa idade (CONSELHO NACIONAL DE SAÚDE, 2014).

A formulação PPSV23 atual contém os seguintes sorotipos capsulares: 1, 2, 3, 4, 5, 6B, 7F, 8, 9N, 9V, $10 \mathrm{~A}, 11 \mathrm{~A}, 12 \mathrm{~F}, 14,15 \mathrm{~B}, 17 \mathrm{~F}, 18 \mathrm{C}, 19 \mathrm{~F}, 19 \mathrm{~A}, 20,22 \mathrm{~F}, 23 \mathrm{~F}$ e 33F. É fornecido como uma dose única de 0,5 $\mathrm{mL}$ ou um frasco multidose de $5,0 \mathrm{~mL}$ para ser administrado por via intramuscular ou subcutânea no músculo deltoide ou na parte média da coxa lateral. Os efeitos colaterais comuns incluem reações leves no local da injeção (por exemplo, inchaço), dor de cabeça, fadiga e mialgia PNEUMOCOCCAL VACCINE POLYVALENT (PNEUMOVAX23), 2011).

Há evidências de que a vacina PPSV23 é efetiva contra a DPI em adultos e que provavelmente também reduz a incidência da PAC. Duas revisões sistemáticas, sendo uma delas comissionada pela Organização Mundial de Saúde (OMS), foram consistentes em descrever uma proteção contra DPI e pneumonia por qualquer causa em adultos jovens e saudáveis e uma menor extensão de proteção contra a DPI na população geral de idosos (MOBERLEY S, et al., 2013).

A vacina PPSV23 tem o objetivo de melhorar a resposta imunológica T-independente, caracterizando uma curta duração, e ao ser utilizada em crianças em idades abaixo de 2 anos, não induz a memória imunológica devido a imaturidade do sistema imunológico. Entretanto, em idade superior a mencionada, o efeito da vacina induz o dobro da produção de anticorpos específicos para os sorotipos alvos da vacina, ao decorrer de 14 a 21 dias (CONSELHO NACIONAL DE SAÚDE, 2014).

É estimado que os níveis de anticorpos regressem aos níveis pré-vacinação em cerca de 5-10 anos após a primeira dose de PPSV23. Contudo, a revacinação só é aconselhada em casos específicos devido à incerteza quanto ao benefício da administração de uma segunda dose devido, principalmente, ao fenómeno "hiporresponsividade" que será explicado mais à frente (REIS SAA, 2015).

Foi concluído que PPSV23 é efetiva contra Doenças Invasivas Pneumocócicas (DIP) e Pneumonia Pneumocócica (PP) em idosos, sendo os valores da eficácia da vacina, derivados da meta-análise de Clínicos Controlados e Aleatórios (RCTs) com baixo risco de viés, $73 \%$ contra DIP e $64 \%$ contra PP (25\% ao incluir estudos com alto risco de viés) (FALKENHORST G, et al., 2017).

Uma revisão sistemática da Colaboração Cochrane também indicou a presença de forte evidência de eficácia da PPSV23 para a prevenção de DPI em adultos em geral com uma redução de chance de $74 \%$ (MOBERLEY S, et al., 2013). Nesse mesmo estudo, pode-se observar uma diminuição na probabilidade de surgimento de pneumonia em relação ao amplo aspecto causal, em torno de $46 \%$ nos países subdesenvolvidos (MARTINS SP, 2019).

Adicionalmente, estudos observacionais sobre a efetividade da vacina PPSV23 mostraram que ela possui efetividade entre 50 e $80 \%$ para a prevenção da DPI em indivíduos adultos portadores de comorbidades, tais como diabetes, insuficiência cardíaca, pneumopatias e insuficiência renal (SCHIFFNER-ROHE J, et al., 2016).

Outro achado relevante entre receptores da vacina que chegaram a desenvolver pneumonia é que sua gravidade e risco de morte se mostraram reduzidos. Estudos observacionais também demonstraram efeito protetor da vacina em indivíduos soropositivos para o HIV com baixa ou moderada imunossupressão (LIANG $\mathrm{Q}$, et al, 2016).

Um estudo realizado em Taiwan demonstrou que o grupo vacinado com PPV23 apresentou menores riscos de desenvolvimento de DIP e, quando a desenvolviam, faziam-na com menor necessidade de hospitalização, menor prevalência de insuficiência respiratória ou menor tempo de internação (TAVARES FS, 2019). Por conseguinte, também com menos ônus aos sistemas de saúde, o que traduz que a vacinação antipneumocócica representa menor custo-efetividade e mortalidade (KUO CS, et al., 2016).

Uma resposta imunológica melhorada após a administração de PPSV23 foi observada em outro estudo usando um intervalo de 4 anos entre as vacinações de PCV13 e PPSV23, em comparação com 30 dias pósvacinação com PCV13 (em adultos com 50 anos) (JACKSON LA, et al., 2013). Outro estudo que avaliou a 
administração de PPSV23 em 8 semanas após o PCV13 (em adultos com idade> 50 anos), as respostas imunológicas para os sorotipos exclusivos do PPSV23 foram superiores, enquanto as respostas aos sorotipos compartilhados foram não inferiores na Semana 12, em comparação com o PCV13 administrado isoladamente (BUCHWALD U, et al., 2020)

É importante notar que a PPSV23 é considerada uma vacina custo-efetiva. Por exemplo, Jiang Y, et al. (2014) compararam através de modelos matemáticos a vacinação com a PPSV23 com a ausência de vacinação no contexto do Reino Unido e estimaram uma Razão de Custo-Efetividade Incremental (RCEI) de $£ 8.413(\mathrm{R} \$ 41.055,44)$ por Ano de Vida Ajustado por Qualidade.

O comitê estratégico de especialistas em imunização da OMS (SAGE) recomenda o uso da vacina PPSV23 para idosos e adultos portadores de doenças de alto risco para infecções pneumocócicas e discorre sobre a necessidade do aumento da cobertura vacinal nessa população alvo (OMS, 2021).

Atualmente, no Brasil, dispomos nos Centros de Referência para Imunobiológicos Especiais (CRIEs) de duas vacinas pneumocócicas oferecidas de forma gratuita à população com as indicações específicas, a 23valente (PPSV23) e a 10-valente (conjugada-Pnc10). Os 23 tipos capsulares de pneumococos incluídos na vacina são: 1, 2, 3, 4, 5, 6B, 7F, 8, 9N, 9V, 10A, 11A, 12F, 14, 15B, 17F, 18C, 19A, 19F, 20, 22F,23F e 33F (MONTEIRO CN, et al., 2015).

De acordo com o PNI e a $4^{a}$ edição do manual dos CRIEs, a PPSV23 é indicada para indivíduos com 2 anos ou mais de idade e portadores de ao menos uma das condições clínicas (Quadro 1) (MINISTÉRIO DA SAÚDE, 2014).

Quadro 1 - Condição clínica para PPSV23 segundo o PNI.

\begin{tabular}{|c|c|}
\hline $\begin{array}{c}\text { Asma persistente, moderada ou grave } \\
\text { relacionadas }\end{array}$ & Implante de Cóclea \\
\hline Cardiopatias crônicas & $\begin{array}{c}\text { Imunodeficiências congênitas } \\
\text { Diabetes Mellitus }\end{array}$ \\
\hline $\begin{array}{c}\text { Nefropatia crônica / hemodiálise / síndrome } \\
\text { nefrótica }\end{array}$ \\
\hline Doença neurológica incapacitante & $\begin{array}{c}\text { Transplantados de células tronco } \\
\text { hematopoiéticas }\end{array}$ \\
\hline Fibrose Cística & Transplantados de órgãos sólidos \\
\hline Fístula liquórica & Trissomias \\
\hline Hepatopatias Crônicas & Doenças de depósito \\
\hline HIV / Aids & $\begin{array}{c}\text { Pneumopatia crônica, exceto asma } \\
\text { intermitente ou persistente leve }\end{array}$ \\
\hline
\end{tabular}

Fonte: Ferreira LDS, et al. (2021); dados extraídos do Ministério da Saúde (2014).

Além das indicações clínicas listadas acima, o PNI também prevê a aplicação da PPSV23 para pessoas a partir dos 60 anos de idade, quando hospitalizadas ou residentes em instituições fechadas, como asilos, casas geriátricas, casas de repouso e povos indígenas (MINISTÉRIO DA SAÚDE, 2014).

Quando comparamos as indicações de dispensação da PPSV23 de acordo com o PNI com as recomendações do Comitê de Aconselhamento sobre Práticas de imunização norte-americano (ACIP), observa-se que no Brasil o PNI não prevê a vacinação com PPSV23 para os indivíduos que não sejam tabagistas e/ou etilistas crônicos, ou para os idosos sem comorbidades (PALLOTTA A e REHM SJ, 2016).

Ouros estudos apontam que $12 \%$ da população acima dos 40 anos de idade possuam Doença Pulmonar Obstrutiva Crônica (DPOC), essa estimativa e como base em cidades com população entre 100.000 a 120.000 habitantes, levando em consideração que não haja superposição de indivíduos enfermos com diabetes mellitus e DPOC, seria cerca de 12.600 pessoas indicas para a vacinação com PPSV23 (SEADE, 2019). 
É perceptível que, muitos outros países confrontam com situação parecidas, onde uma baixa frequência de vacinação pneumocócica se faça presente na população, principalmente atrelado aos indivíduos com indicação clínica da vacina. É fundamental frisar, que a criação de estratégias para favorecer o alcance da cobertura vacinal se faz necessário, não somente para vasto número de imunobiológicos (GONZÁLEZ DFR, et al., et al., 2015).

No panorama brasileiro, os problemas inerentes à produção e à distribuição da PPSV23, possuem causas bem fundamentadas, uma delas e de que pessoas com indicação clínica de receber a vacina de acordo com a PNI, acabam não sendo vacinados. Uma das causas e que, existe falta de conhecimento dos profissionais área da saúde e da população sobre a PPSV23, agravado pela forma que o sistema atual distribui a vacina. Nacionalmente, a dispersão é toda concentrada nos CRIEs, fator esse que colabora veementemente com o quadro situacional alarmante. Outro impasse significante ligado à deficiência de conhecimento sobre a PPSV23 no meio profissional (Médicos), está em volta do não acreditar, de uma grande parcela desses profissionais, que os mesmos possam indicar e prescrever essa vacina (MARTINS SP, 2019).

De certo modo, a existência de uma barreira extra para o tratamento das doenças pneumocócicas é a resistência adquirida pelo $S$. pneumoniae a antibióticos, portanto, vacinar pessoas acima de 65 anos de idade é essencial, sendo considerada a forma mais eficaz e eficiente de prevenção (PAPADATOU I e SPOULOU V, 2016).

\section{CONSIDERAÇÕES FINAIS}

As doenças pneumocócicas causam grandes impactos econômicos na sociedade, pelo morbimortalidade gerada, além de afetar o sistema de saúde mundial, e principalmente no Brasil que possui uma certa limitação evidente no âmbito da saúde pública. Dessa forma, esse problema se agrava ainda mais, quando surgem cepas resistentes a antibióticos comumente utilizados no combate a infecção por $S$. pneumoniae. Então, o uso da vacina PPSV23, conforme supracitado no estudo, pode conter o impacto crescente da doença, e também reduzir a resistência aos antibióticos, evitando o surgimento de cepas resistentes, pelo uso diminuto desses antibióticos. Por fim, faz-se necessário mais estudos desse cunho, para que se possa reunir informações e associá-la, de maneira a melhor compreender os impactos que a vacinação contra as doenças pneumocócicas possa trazer para a sociedade.

\section{AGRADECIMENTOS E FINANCIAMENTO}

Agradecimentos a Escola Superior da Amazônia (ESAMAZ), pelo suporte técnico disponibilizado na elaboração desse estudo.

\section{REFERÊNCIAS}

1. ALEXANDRE RF, et al. Avaliação de custo-efetividade da vacina pneumocócica conjugada 13-valente comparada à vacina pneumocócica polissacarídica e não vacinação no Sistema de Saúde Suplementar Brasileiro. J Bras Econ Saúde, 2016; 8(3): 192-196.

2. ALVAREZ CE, et al. Survey of vaccination practices in patients with diabetes: A report examining patient and provider perceptions and barriers. Journal of Clinical \& Translational Endocrinology, 2017; 1(9): 15-17.

3. AMERICAN DIABETES ASSOCIATION. Improving Care and Promoting Health in Populations: Standards of Medical Care in Diabetes - 2019. Diabetes Care. 2019; 1(42): 7-12.

4. BAHIA L, et al. Systematic review of pneumococcal disease costs and productivity loss studies in Latin America and the Caribbean. Vaccine, 2013; 31: 33-44.

5. BLAMEY, R. Vacunas anti-neumocóccicas en adultos: actualización. Revista chilena de infectología, 2014;31(5): 607609.

6. BUCHWALD U, et al. Sequential administration of Prevnar 13 and PNEUMOVAX23 in healthy participants 50 years of age and older. 2020. In Presented at: 12th International Symposium on Pneumococci and Pneumococcal Disease (ISPPD), 2020; 1; 20-24.

7. CAIERAO J, et al. Serotypes and genotypes of invasive Streptococcus pneumoniae before and after PCV10 implementation in Southern Brazil. PLoS One, 2014; 9(10): e111129.

8. $\mathrm{CHEN} \mathrm{CH}$, et al. Vaccination coverage and associated factors for receipt of the 23-valent pneumococcal polysaccharide vaccine in Taiwan: a nation-wide community-based study. Medicine 2018; 97 : e9773. 
9. CONSELHO NACIONAL RE Resoluções do Conselho Nacional de Saúde. Brasília, DF: Editora MS, 2014.

10. CORREIA S. Vacinação anti-pneumocócica no idoso. Rev Port Med Geral Fam, 2013; (1): 386-393.

11. COSTA RP, et al. A doença pneumocócica e recomendações GRESP para a vacinação antipneumocócica na população adulta. 2016; 70-74.

12. FALKENHORST G, et al. Effectiveness of the 23-Valent Pneumococcal Polysaccharide Vaccine (PPV23) against Pneumococcal Disease in the Elderly: Systematic Review and Meta-Analysis. 2017; 12 (1): e0169368.

13. FERKOL T, SCHRAUFNAGEL D. The global burden of respiratory disease. Ann Am Thorac Soc. 2014; $11(3)$ : $404-6$.

14. GATWOOD J, et al. Pneumococcal vaccination in older adults: an initial analysis of social determinants of health and vaccine uptake. Vaccine 2020; 38: 5607-17.

15. GONZÁLEZ DFR. O Envelhecimento e a Autoimunidade. Faculdade de Medicina, Universidade de Coimbra, Portugal. $2015 ; 1-78$.

16. HUIJTS SM, et al. Post-hoc analysis of a randomized controlled trial: Diabetes mellitus modifies the efficacyf the 13valent pneumococcal conjugate vaccine in elderly. Vaccine. 2017; 35(34): 4444-4449.

17. JACKSON LA, et al. Influence of initial vaccination with 13-valent pneumococcal conjugate vaccine or 23-valent pneumococcal polysaccharide vaccine on antipneumococcal responses following subsequent pneumococcal vaccination in adults 50 years and older. Vaccine, 2013; 31: 3594-602.

18. JIANG Y, et al. Cost-effectiveness of vaccinating the elderly and at-risk adults with the 23-valent pneumococcal polyssacharide vaccine or 13-valent pneumococcal conjugate vaccine in the UK. Expert Rev Pharmacoecon Outcomes Res, 2014: 913-927.

19. KUO CS, et at. Effectiveness of 23-valent pneumococcal polysaccharide vaccine on diabetic elderly. Medicine (Baltimore). 2016; 95(26): 4064.

20. LIANG Q, et at. Vaccine profile of PPV23: Beijing Minhai Biotech 23-valent pneumococcal vaccine. Expert Review of Vaccines, 2016; 4: 1-9.

21. MARQUES SC, et al. A importância da vacinação dos adultos com diabetes tipo 2 na prevenção da doença invasiva pneumocócica. Rev Port Endocrinol Diabetes Metab. 2016; 11(1): 60-68.

22. MARTINS JP. Estratégias para otimizar o acesso à vacina pneumocócica polissacarídica 23-valente junto à população de adultos com indicação clínica no sus. Universidade Estadual Paulisa. 2019; 1: 1-23.

23. MINISTÉRIO DA SAÚDE. Manual de Normas e Procedimentos para Vacinação. 2014. Disponível em: https://portaldeboaspraticas.iff.fiocruz.br/biblioteca/manual-de-normas-e-procedimentos-para-vacinacao/. Acessado em: 28 de setembro de 2021.

24. MIRANDA JMD. Infecção Pneumocócica Invasiva no Idoso em Portugal em 2008 e 2009. Universidade de Lisboa Faculdade de Ciências. 2011; 1-50.

25. MOBERLEY S, et al. Vaccines for preventing pneumococcal infection in adults. Cochrane Database of Systematic Reviews. Chichester, UK: John Wiley \& Sons, Ltd, 2013; 1: 1-66.

26. MONTEIRO CN, et al. Coverage by the public health services of medication and vaccines for the population with diabetes mellitus. Ciênc. saúde coletiva, Rio de Janeiro, 2015, 20(2): 557-64.

27. MURAKAMI Y, et al. A nationwide survey of PPSV23 vaccine coverage rates and their related factors among the elderly in Japan, 2016. Nihon Koshu Eisei Zasshi, 2018; 65: 20-4.

28. NUNES BP, et al. Hospitalização em idosos: associação com multimorbidade, atenção básica e plano de saúde. Rev Saude Publica. 2017; 51: 1-43.

29. PALLOTTA A, REHM SJ. Navigating pneumococcal vaccination in adults. Cleveland Clinic Journal of Medicine, 2016; 83(6): 427-433.

30. PAPADATOU I, SPOULOU V. Pneumococcal Vaccination in High-Risk Individuals: Are We Doing It. 2016; 23(5): 388395.

31. PICHICHERO ME, et al. Next generation protein based Streptococcus pneumoniae vaccines, 2016; 12(1): $194-205$.

32. PNEUMOCOCCAL VACCINE POLYVALENT (PNEUMOVAX23). Whitehouse Station, NJ: Merck \& Co., Inc., 2011. Disponível em:<https://www.fda.gov/vaccines-blood-biologics/vaccines/pneumovax-23-pneumococcal-vaccinepolyvalent>. Acesso em: 30/09/2021.

33. REIS SAA. A importância da vacinação no idoso. Faculdade de medicina da universidade de coimbra, $2015 ; 1-67$.

34. SCHIFFNER-ROHE J, et al. Efficacy of PPV23 in Preventing Pneumococcal Pneumonia in Adults at Increased Risk A Systematic Review and Meta-Analysis. PLOS ONE, 2016; 11(1): e0146338.

35. FUNDAÇÃO SISTEMA ESTADUAL DE ANALISE DE DADOS (SEADE). Informações dos Municípios Paulistas. 2019. Disponível em: http://www.imp.seade.gov.br/frontend/\#/. Acesso em: 29 de setembro de 2019.

36. TAVARES FS, et al. Situação vacinal antipneumocócica em pacientes com diabetes atendidos em hospital de referência no Distrito Federal, Brasil. Revista Eletrônica Acervo Saúde, 2019; 11(13): e1282.

37. VILA-CORCOLES A, OCHOA-GONDOR O. Preventing pneumococcal disease in the elderly: recent advances in vaccines and implication for clinical practice. Drugs \& Aging.2013; 30: 263-276.

38. ORGANIZAÇÃO MUNDIAL DA SAÚDE (OMS). Doença pneumocócica. 2017. Disponível em: https://www.who.int/ith/diseases/pneumococcal/en/. Acessado em: 30 de março de 2021.

39. ZINTGRAFF J, et al. Distribution of PCV13 and PPSV23 Streptococcus pneumoniae serotypes in Argentinean adults with invasive disease, 2013---2017. Revista Argentina de Microbiogía, 2020; 52(3): 189-194. 